\title{
Ist es an der Zeit, die Aktionärsrechte zu stärken?
}

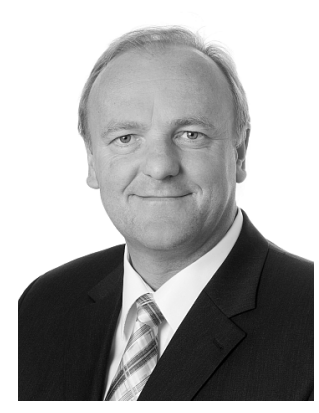

Egon Franck

Corporate Governance, Abzockerinitiative, Aktionärsrechte, Residualansprüche, Interessenkonflikte, Board Primacy

Corporate governance, shareholder rights, residual claims, conflicts of interest, board primacy

Forderungen nach mehr Aktionärsdemokratie finden zunehmende gesellschaftliche und auch akademische Akzeptanz. Der Beitrag erläutert, weswegen sie dennoch falsch sind, und empfiehlt eine Rückbesinnung auf die Kernelemente der Board Primacy im Falle anste-

hender Aktienrechtsrevisionen.

The idea of empowering the shareholder gains momentum in the political as well as in the academic corporate governance discussion. The paper explains why the concept of a shareholder democracy is not a viable governance mechanism in this context. Future reforms of corporate governance regulations should reconsider the basic principles of board primacy.

\section{Ausgangslage und Problemstellung ${ }^{1}$}

Finanzskandale und Salärexzesse bei grossen Aktiengesellschaften - man denke stellvertretend an die Berichterstattung über Enron, WorldCom oder Lehman - lassen den Eindruck entstehen, dass die so genannte Corporate Governance nur sehr fehlerhaft funktioniere. Die Ansicht, dass „endlich etwas getan werden muss“, um die als defekt empfundene Überwachungsmechanik grosser Aktiengesellschaften zu reparieren, ist weit verbreitet. Immer mehr Beteiligte an der Debatte glauben, dass eine Stärkung der Aktionärsrechte die richtige „Reparaturmassnahme" sei.

Stellvertretend sei die in der Schweiz intensiv diskutierte, so genannte Abzockerinitiative des Unternehmers Thomas Minder genannt, die insgesamt 24 Forderungen an ein neues Aktienrecht formuliert (Initiativkomitee 2011). Aber auch in der akademischen Corporate Governance-Diskussion, die im Schnittfeld zwischen Wirtschafts- und Rechtswissenschaften geführt wird, findet die Forderung nach einer „Aufrüstung“ der Aktionäre mehr und mehr Fürsprecher (Bebchuk 2005). ${ }^{2}$

Vor diesem Hintergrund soll der Frage nachgegangen werden, ob es wirklich an der Zeit ist, die Aktionärsrechte zu stärken.

1 Der Beitrag entspricht weitgehend meiner Rede anlässlich des Dies academicus der Universität Zürich am 30. April 2011; vgl. Franck (2011).

2 „The Case for Increasing Shareholder Power“ heisst folgerichtig ein im Harvard Law Review 2005 erschienener Artikel eines der prominentesten Teilnehmer an der Debatte: Lucian A. Bebchuk. 


\section{In welchem Sinne sind Aktionäre „schwach“ und was bedeutet die Stärkung der Aktionärsrechte?}

Der Einfluss der Aktionäre auf unternehmerische Entscheidungen kann einerseits gering sein, weil sie ihre Rechte aufgrund eines Problems kollektiven Handelns nicht wirklich ausüben. In der Bildersprache könnte man von einem „feststeckenden Schwert“ der Aktionäre sprechen.

Der Einfluss der Aktionäre kann aber andererseits auch in einem materiellen Sinne gering sein, weil die Anzahl und der Inhalt der Punkte, über die sie in der Generalversammlung abstimmen dürfen, durch den rechtlichen Rahmen sehr eng gefasst wird. Bildlich könnte man dies als Problem des „stumpfen Schwertes“ der Aktionäre umschreiben.

\subsection{Das Problem kollektiven Handelns in der Modern Corporation}

Stellen wir uns Aktionäre vor, die jeweils nur Kleinstanteile an der so genannten Modern Corporation, der Aktiengesellschaft im Streubesitz, halten. Damit sie aktiv an der Entscheidungsfindung der Unternehmung teilnehmen, müssten sie sich über Chancen und Risiken des unternehmerischen Handelns informieren, eventuell Gegenvorschläge formulieren und dafür Mehrheiten organisieren. Dies verursacht jedoch private Kosten, die der einzelne Aktionär dem erwarteten Nutzen gegenüberstellt. Durch seinen Kleinstanteil kann der Aktionär aber nur in einem geringen Ausmass von eventuell erzielbaren besseren Unternehmensergebnissen profitieren. „Rational apathische“ (Bainbridge 2006, 1745) Kleinaktionäre werden sich daher nicht organisieren, um ihre Rechte aktiv wahrzunehmen.

Der Aktiengesellschaft im Streubesitz ist jedoch die empirische Relevanz abhanden gekommen. In Europa werden Aktiengesellschaften meist durch grosse Blockhalter dominiert. Aktuell zeigen dies etwa Fauver und Fürst (2006) anhand ihres Samples, das 786 Aktiengesellschaften aus der grössten europäischen Volkswirtschaft, Deutschland, enthält: In 53\% aller Fälle hält der grösste Aktionär mindestens 25\% der Stimmen, in 39\% aller Fälle sogar mehr als 50\%. In der Schweiz ist der Anteil der börsenkotierten Firmen, bei welcher der grösste Aktionär mehr als 50\% der Stimmen besitzt, sogar noch leicht höher, nämlich 42\% im Jahr 2009 (Stadelmann 2010, 29). In den USA setzte vor allem seit 1980 eine starke Konzentrationstendenz in den Eigentumsstrukturen grosser Aktiengesellschaften ein. ${ }^{3}$ Schon 1990 zog Black (1990, 574) wie folgt Bilanz:

„... the model of public companies as owned by thousands of anonymous shareholders simply isn't true. There is a limited number of large shareholders, and they know each other."

In einer ganz aktuellen Untersuchung bestätigt Davis (2009, 33) dieses Bild:

„... nearly three-quarters of the average Fortune 1000 corporation's shares were owned by institutional investors, with mutual funds making up the most concentrated block."

3 Bhagat et al. (2004, 2), die diese Eigentumsstrukturen über die Periode 1983-1993 in den USA untersuchten, berichten über einen „... significant secular increase in large-block shareholding... with sharp percentage increases in these holdings by mutual funds, partnerships, investment advisors, and employee benefit plans". 


\section{2 „Schwache“ Aktionäre aufgrund einer rechtlich verankerten Board Primacy}

Im Vergleich zum Kleinaktionär partizipiert ein Blockhalter in viel höherem Umfang an den positiven Folgen besserer unternehmerischer Entscheidungen, denn Kurs- und Dividendensteigerungen schlagen auf jede einzelne seiner Aktien zu Buche. Da Blockhalter bei gleichen Kosten der Informationsaktivität einen viel höheren Nutzen haben, lohnt es sich für sie, das Unternehmensgeschehen aktiv zu verfolgen und mit zu gestalten. Wenn Blockhalter ihre „Schwäche“ beklagen, hat dies vor allem mit der materiellen Ausgestaltung der Aktionärsrechte zu tun.

Unabhängig von gegebenen nationalen Regulierungsunterschieden ist das „Schwert“ der Aktionäre in allen entwickelten Volkswirtschaften tatsächlich erstaunlich „stumpf“. Das Gesetz weist nämlich unmissverständlich dem Board of Directors - dem Verwaltungsrat die zentrale Entscheidungsgewalt in der Aktiengesellschaft zu. In der Schweiz beispielsweise enthält Art. 716a Abs. 1 OR eine abschliessende Liste von unübertragbaren und unentziehbaren Aufgaben, die vom Verwaltungsgrat als Gesamtorgan zu erfüllen sind. Diese Aufgaben dürfen dem Verwaltungsrat auch nicht durch die Generalversammlung entzogen oder gar an diese „rückdelegiert“ werden. Zu den zwingenden Führungsaufgaben des Verwaltungsrats gehören unter anderem die Oberleitung der Gesellschaft und Erteilung der nötigen Weisungen, die Festlegung der Organisation, die Wahrnehmung der Finanzverantwortung und die Bestellung und Beaufsichtigung der Geschäftsführung. In den USA ist die Situation ähnlich. ${ }^{4}$ Bereits 1918 stellte ein New Yorker Gericht klar, dass die entsprechende Macht des Boards „original and undelegated“ (zitiert nach Bainbridge 2006, 1756) sei.

Das Aktienrecht erschafft eine klare Befehlsgewalt in der Firma und erkennt die Autorität zu ihrer Ausübung eindeutig dem Board zu. Zwar existiert ein Netzwerk von Mechanismen der Rechenschaftsablage, aber es wird darauf geachtet, dass dieses die Autorität des Boards nicht untergräbt (vgl. dazu ausführlich Bainbridge 2006, 1747). Genau in diesem Kontext ist beispielsweise die systematische Begrenzung der Haftungsansprüche gegen Boardmitglieder zu sehen. In den USA (und übrigens auch in der schweizerischen und deutschen Gerichtspraxis) kommt dabei die so genannte „Business Judgment Rule“ zum Einsatz. Wenn ein Direktor bei einer unternehmerischen Entscheidung vernünftigerweise annehmen durfte, auf der Grundlage angemessener Information zum Wohle der Firma zu handeln, kann ihm keine Pflichtverletzung vorgeworfen werden. Der Schutz der Direktoren zur Wahrung ihrer Autorität ist das erklärte Ziel dieser Regel. Daran lässt z. B. das Oberste Gericht von Delaware - einem der beliebtesten Inkorporationsstaaten der USA - keinen Zweifel (zitiert nach Bainbridge 2006, 1747):

„The business judgment rule exists to protect and promote the full and free exercise of the managerial power granted to the Delaware directors. "

\subsection{Stärkung der Aktionärsrechte als „rechtliches Wendemanöver“ von der Board Primacy zur Shareholder Primacy}

Die Aktivisten des Shareholder Empowerments sehen den von den gegenwärtigen rechtlichen Rahmenbedingungen gesetzten Pfeiler der Board Primacy nicht als legitim an. Sie fordern

4 Hans Caspar von der Crone hat mich darauf hingewiesen, dass Art. 716a OR sogar noch weiter geht als die vergleichbaren Bestimmungen des ausländischen Rechts, die zwar die Kompetenz des Boards ebenso betonen, aber mehr Raum für eine Modifikation der Statuten bieten. Insofern ist die Schweiz ein „Musterfall“ der Board Primacy. 
stattdessen die Durchsetzung des Konzeptes einer Shareholder Primacy in der Corporate Governance und damit ein genuines „rechtliches Wendemanöver“. Die Befehlsgewalt des Boards sollte nicht wie heute direkt aus der Rechtsform der Aktiengesellschaft entstehen, sondern nur indirekt aus einer Delegation von Entscheidungsrechten durch die Aktionäre abgeleitet werden können. Eine „abgeleitete“ Befehlsgewalt, welche Aktionäre an Direktoren lediglich „verleihen“, können sie diesen nämlich auch jederzeit wieder entziehen.

Um dem Modell der delegierten Befehlsgewalt Gültigkeit zu verschaffen, fordern die Aktivisten der Shareholder Primacy eine erhebliche rechtliche „Aufrüstung“ der Aktionäre. Grundsätzlich gehen diese „Aufrüstungswünsche“ in drei Richtungen (vgl. z. B. Bratton/ Wachter 2010, 671): Erstens geht es um Reformen des Wahlsystems für Boardmitglieder mit dem Ziel, Direktoren viel schneller und leichter aus dem Amt entfernen zu können. ${ }^{5}$ Zweitens sollen Statutenänderungen auch gegen den Willen des Verwaltungsrats in die Tat umgesetzt werden können, mit dem Ziel einer direkten Einflussnahme auf bestimmte Bereiche der Geschäftspolitik. Bebchuk (2006) spricht von der so genannten „shareholder power over rulesof-the-game decisions“. Diese reichen von der Aufbauorganisation des Verwaltungsrats, über die Festlegung der Dividendenhöhe bis zur Re-Inkorporierung der Gesellschaft in einem anderen Bundesstaat (vgl. dazu ausführlich z. B. Bratton/Wachter 2010, 672-673). Bratton/Wachter (2010, 673) bemerken über den Trend dieser Forderungen ganz zu Recht:

„... we note an implicit open end: once any door to the reversal of board business judgment is opened, there will be no principled basis for containing shareholder mandates respecting business policy. "

Die dritte Richtung der gewünschten „Aufrüstung“ bilden schliesslich die so genannten „Say on pay“-Initiativen. Dabei geht es nicht etwa um eine Information der Aktionäre über Kompensationspakete, sondern um die vorgängige Genehmigung der Saläre des Boards und des Topmanagements direkt durch die Aktionäre auf einer mandatierten jährlichen Basis. ${ }^{6}$

\section{Theoretische Grundlage des Shareholder Primacy Paradigmas}

Die theoretische Grundlage für das Shareholder Primacy Konzept findet man in einer hauptsächlich von Ökonomen geprägten Denkschule, aus der wenige Bausteine skizziert werden sollen. ${ }^{7}$

\subsection{Die Rolle so genannter Residualansprüche}

Entlang einer vor allem auf Jensen/Meckling (1976), Fama/Jensen (1983a, 1983b) und Jensen (2000) zurückgehenden Betrachtungsweise entsteht eine Firma, indem verschiedene Akteure ihre Beiträge für einen Produktionsprozess „zusammenlegen“ (vgl. zu diesen und den

5 Auch die Minder-Initiative in der Schweiz fokussiert stark auf eine derartige Reform des Wahlsystems. Dies offenbart sich z. B. in Forderungen nach jährlicher Einzelwahl der Mitglieder des VR, des VR-Präsidenten, der Mitglieder des Vergütungsausschusses und des unabhängigen Stimmrechtsvertreters. Ebenso fordert die Initiative keine Organstimmrechtsvertretung, keine Depotstimmrechtsvertretung, elektronische Fernabstimmung, Stimmzwang der Pensionskassen im Interesse ihrer Versicherten und Offenlegung der Abstimmung der Pensionskassen.

6 Im Rahmen der Minder-Initiative sind die Abstimmung der Generalversammlung über die Gesamtsumme der Vergütungen des Verwaltungsrats und der Geschäftsleitung ebenfalls zentrale Forderungen.

7 Eine ausführliche und viel weiter gehende Auseinandersetzung mit den unterschiedlichen Legitimationsgrundlagen so genannter Stakeholderansprüche liefert Brink (2011) in einem gerade erschienenen, sehr lesenswerten Beitrag, der dem Autor zum Zeitpunkt der Manuskripterstellung noch nicht vorlag. 
folgenden Überlegungen auch Franck 2002). Arbeiter verschiedener Ausbildung liefern spezialisierte Fähigkeiten, Arbeitskraft und -zeit, die öffentliche Hand liefert Infrastrukturen, Geldgeber stellen Kapital für Investitionen bereit usw. Die verschiedenen Ressourcen werden deswegen zusammengelegt, weil gegenüber ihrer isolierten Verwendung ein Mehrwert entsteht.

Prinzipiell können nun alle bis auf eine Partei vertraglich festgelegte Ansprüche auf den erzeugten Produktionswert eingeräumt bekommen. So erhalten z. B. die meisten Arbeitslieferanten vertraglich vereinbarte Löhne und die Fremdkapitalgeber werden durch vertraglich geregelte Ansprüche auf Rückzahlungen und Zinsen entschädigt. Zieht man alle vorher auf diese Weise in Verträgen vereinbarten Ansprüche von dem erwirtschafteten Produktionswert ab, so offenbart sich das so genannte Residuum als eine Restgrösse.

Mindestens eine Partei, die Inputs eingebracht hat, muss als Entlohnung mit dieser Restgrösse, dem Residuum, vorlieb nehmen. In einer Welt mit unvollständiger Information können nachträglich immer Entwicklungen eintreten, die den Produktionswert beeinflussen, ohne dass in den abgeschlossenen Verträgen dafür Reaktionen vorgesehen wurden. Der Residualanspruchshalter „versichert“ also in gewisser Weise alle anderen Inputlieferanten, weil er die finanziellen Folgen „,nicht vorhergesehener“ Ereignisse in seinem „Restanspruch“ auffängt.

\subsection{Versicherung durch Aktionäre und die daraus folgende „separation of ownership and control“}

Nun kommt der entscheidende „Kunstgriff“ dieser Denkschule: Sie sieht für die Rolle der „Restanspruchshalter“ ausschliesslich die Aktionäre vor und erklärt damit gleichzeitig alle anderen Parteien zu Haltern vertraglich fixierter Ansprüche. Aktionäre sind prädestiniert für die Übernahme „übrig gebliebenen“ Risikos, weil sie dieses Risiko in Kleinstportionen übernehmen, an der Börse weiter handeln und über ein Anlagenportfolio diversifizieren können.

Diese Form der Risikobewirtschaftung setzt aber automatisch die Delegation der Entscheidungen über den Ressourceneinsatz in der Unternehmung an Spezialisten, die Direktoren, voraus. Ansonsten müsste die Unternehmung durch beliebig viele und zudem mit jedem Börsendeal jederzeit austauschbare Aktionäre geführt werden - eine Vorstellung, die nicht umsetzbar ist. Die resultierende „separation of ownership and control“ (Berle/Means 1932) hat nun aber eine wichtige Folge: Die einzige schädigbare Partei in dieser Sicht der Dinge die Aktionäre, die keinen Vertrag mit fixierten Ansprüchen halten - fällen die Entscheidungen über den Ressourceneinsatz in der Firma - also die Entscheidungen mit Schädigungspotential - nicht mehr selbst. Eine dritte Partei, die Direktoren, fällen diese stattdessen.

Wem sollten die Direktoren verantwortlich sein? Das ist offensichtlich: Den einzigen Haltern von Residualansprüchen, den Aktionären, weil nur sie zu „Opfern“ werden können. Von allen Beteiligten haben nur sie sich auf einen unbestimmten Rest verpflichtet, der von den Direktoren manipulierbar ist. Die Shareholder Primacy folgt zwingend aus dieser Theorie. Über die Corporate Governance ist den „ausgelieferten“ Aktionären eine Art „verlängerter Arm" zu bauen, damit sie den Konsum des Shareholder-Value durch die Direktoren wirksam verhindern können. 


\section{Firmenspezifische Investitionen und viele „Schutzlose“}

Eine realistischere Theorie der Firma berücksichtigt, dass verschiedene Akteure illiquide, so genannte „firmenspezifische“ Investitionen tätigen können (Stout 2007, 795-796). Mitarbeiter, Lieferanten, Kreditgeber, Kunden und andere Stakeholder investieren Zeit, Mühe, Wissen und Geld in ihre Beziehung mit der Firma. Diese Investitionen sind nur dann von Wert, wenn die Firma nicht untergeht und die Beziehung von Dauer ist.

\subsection{Mitarbeiter als Halter von Residualansprüchen}

Die Unvollständigkeit von Arbeitsverträgen betonend, weist eine ganze Reihe von Autoren darauf hin, dass sich die Rolle der Mitarbeiter, die in so genanntes spezifisches Humankapital investieren, von jener der Aktionäre kaum unterscheidet (vgl. z. B. Furubotn 1988; Hansmann 1993; Blair 1995, 1999; Blair/Stout 1999). Spezifisches Humankapital bezieht sich auf Fertigkeiten, Fähigkeiten, Routinen und persönliche Beziehungen, die nur in dem Unternehmen produktiv verwertbar sind, in dem sie erworben wurden. Ein Indiz für die Existenz unternehmensspezifischen Humankapitals ist z. B., dass Mitarbeiter bei einem Wiedereinstieg in anderen Unternehmen nach einer unverschuldeten Entlassung (etwa aufgrund einer Werksschliessung) empirisch nachgewiesen signifikante und relativ dauerhafte Gehaltsabschläge hinnehmen müssen. ${ }^{8}$

In dieser vor allem von Blair $(1995,1996,1999)$ propagierten Denkrichtung sind Mitarbeiter in Bezug auf ihre Investitionen in spezifisches Humankapital im gleichen Sinne Risikoträger wie die Aktionäre (vgl. die ausführliche Analyse bei Brink 2011, 56-57). Dies zeigt sich, wenn Unternehmen unter finanziellen Druck geraten und Mitarbeiter ihre Entschädigungsansprüche nach unten korrigieren müssen. Soweit der Lohnanspruch der Mitarbeiter ihre unternehmensspezifischen Fähigkeiten betrifft, bezieht er sich also offenbar ebenfalls auf das Residuum, das nach Abzug aller besser durchsetzbaren gesetzlichen und vertraglichen Verpflichtungen im Unternehmen anfällt. Mitarbeiter teilen somit auch das Risiko, dem dieses Residuum ausgesetzt ist.

\subsection{Der Verteilungskonflikt und die Frage der „Aufrüstungssymmetrie“}

Folgt man dieser Denkweise (vgl. insbesondere Blair 1995, 1996, 1999) weiter, landet man automatisch bei einem Verteilungskonflikt. Aktienkapital kann vom Aktionär der Firma, der es gewährt wurde, nicht mehr entzogen werden. Zwar kann der Aktionär sein Wertpapier an der Börse verkaufen. Bereichern sich aber die Manager und Mitarbeiter des Unternehmens durch überhöhte Löhne, dann preist dieser Markt die schlechter gewordenen Gewinnerwartungen umgehend ein. Der Aktionär kommt durch Aktienverkauf aus seiner Investition heraus, trägt aber ein Verlustrisiko. Daher gilt seine Investition als relativ schutzlos den unternehmensinternen Residualeigentümern ausgeliefert.

Mitarbeiter, die in unternehmensspezifisches Humankapital investieren, sind ebenfalls schutzlos. Die mit dem Unternehmen geschlossenen Verträge sind zwangsläufig unvollständig und einen Markt für unternehmensspezifische Fähigkeiten kann es per Definition nicht geben.

8 Blair (1999) schätzt diese Gehaltsabschläge auf der Grundlage empirischer Studien von Topel (1990) und Jacobson et al. (1993) auf durchschnittlich 15-25\%. 
Nun wäre dieser Mangel an Schutz für die Investitionen jeder Seite kein Problem, wenn es keine Anreize gäbe, sich an den Erträgen der Gegenseite zu bereichern. Da beide aus dem Residuum bezahlt werden, das nach Abzug aller gesetzlich und vertraglich besser durchsetzbaren Ansprüche übrig bleibt, konkurrieren Gehaltssteigerungen der Mitarbeiter mit Gewinnsteigerungen der Aktionäre. So beschreibt Blair (1996), dass die Aktienkurse bei Firmen, die interne Sparprogramme einleiteten, regelmässig stiegen. Dagegen wurden Firmen, die es nicht schafften, den Gewerkschaften Lohnkonzessionen abzuringen, durch fallende Aktienkurse „bestraft“ (Blair 1996, 11).

Im Kontext dieses Verteilungskonfliktes wirkt die geforderte Stärkung der Aktionärsrechte wie eine einseitige „Bewaffnung“ der Aktionäre. Als Reaktion könnten Mitarbeiter weniger bereit sein, unternehmensspezifische Fähigkeiten aufzubauen, weil sie systematisch befürchten müssen, nachträglich von erstarkten Shareholdern um ihre Rückflüsse betrogen zu werden (vgl. grundsätzlich zur Beteiligung so genannter Knowledge Worker an der Unternehmensführung Osterloh/Frey 2006). Die Weiterentwicklung der spezifischen Know-how-Basis des Unternehmens durch die Mitarbeiter ist aber ein überlebenswichtiges Anliegen.

Was folgt daraus für die Ausgestaltung der Corporate Governance? Wenn schon eine „Bewaffnung“ der Aktionäre erfolgen soll, dann nur Hand in Hand mit einer ebensolchen der Mitarbeiter. Sollen sie bereit sein, weiterhin als spezifische Investoren tätig zu sein, benötigen sie ebenfalls Mitsprache (vgl. allgemein zur Exit-Voice-Problematik Hirschman 1970; für eine breitere Perspektive der „Employee governance“ siehe Brink 2011, 58). An die Stelle des heute in den meisten Ländern unabhängigen Boards träte nach dieser Sichtweise eine Art Interessenvertreter-Gremium, das aus Delegierten der Aktionäre und Mitarbeiter bestünde.

\section{Teamproduktion und die drohende Eskalation der politischen Rentensuche}

Auf Alchian und Demsetz (1972) geht die Theorie der so genannten Teamproduktion zurück. Unter anderem und sehr vereinfacht weist sie auf Folgendes hin.

Wenn Firmen Güter auf dem Markt verkaufen, ist es meist nicht möglich, genaue Anteile an dem geschaffenen Wert auf den Beitrag einzelner Teammitglieder zurückzurechnen. Wäre der Leistungsbeitrag eines Mitarbeiters exakt separierbar, dann müsste dieser gar nicht erst angestellt werden. Der entsprechende Beitrag könnte dann auch problemlos ausgelagert und über den Markt eingekauft werden. Nur genau jene Leistungsbeiträge bleiben in dieser Sicht der Dinge stets „in der Unternehmung“, für welche diese Verrechnungslogik nicht greift. Die Unternehmung wird also regelrecht definiert durch Teamproduktion und damit durch die Unteilbarkeit des Teamoutputs.

Diese Theorie der Firma legt nahe, dass die Aufteilung des Residuums unter den spezifischen Investoren nicht so einfach über eine „Aufrüstung“ mit Mitspracherechten lösbar ist. Bei dem anstelle des unabhängigen Boards vorgeschlagenen Stakeholder-Gremium haben Mitarbeiter und Aktionäre über ihre Delegierten Mitsprache und damit Macht. Aber die jetzt mächtigen spezifischen Investoren finden angesichts der Unteilbarkeit des Teamoutputs dennoch keine einfach zu legitimierende Teilungsregel. Für jeden Vorschlag über die Aufteilung des Residuums sind stets Alternativen denkbar.

Aus diesem Grund verwandelt sich ein mit Interessenvertretern besetztes Führungsgremium der Aktiengesellschaft immer dann in eine Arena der politischen Rentensuche, wenn die Interessendivergenzen der vertretenen Stakeholder erheblich sind (Anabtawi 2006; Bainbridge 2006; Stout 2007). Diese Gefahr droht, weil neben der bereits beleuchteten Interes- 
sensdivergenz zwischen Aktionären und Mitarbeitern auch die Interessensdivergenzen innerhalb der Shareholder- und Mitarbeitergruppen beachtlich sind.

\subsection{Die Gefahr des Shareholder Sharking 9}

Die Blockhalter-Realität moderner Aktiengesellschaften ist durch erhebliche Interessengräben durchzogen, wie z. B. Anabtawi (2006) ausführlich untersucht hat. Für den einzelnen Blockhalter können nämlich bestimmte Entscheidungen der Aktiengesellschaft über private Umwege Sondererträge abwerfen.

Ein historischer Fall mag zur Illustration realwirtschaftlicher Umwegerträge dienen (Anabtawi 2006, 16-17). 1917 erwarb Du Pont einen Aktienblock bei General Motors mit dem klaren Ziel, das Zuliefergeschäft im Bereich Leder, Polyamid und Farben an sich zu ziehen. Das Vorhaben gelang auch tatsächlich, was aber Mitbewerber bewog, ein AntitrustVerfahren anzustrengen. Der Fall zeigt stellvertretend, dass Grossaktionäre, die als potentielle Zulieferer, Abnehmer, Konkurrenten etc. mit der Unternehmung realwirtschaftlich verflochten sind, diese zur Verfolgung der eigenen Geschäftspolitik missbrauchen können. Die Deformierung der Entscheidungen der Aktiengesellschaft durch solche Blockhalter mit privaten Interessen wird dann z. B. über höhere Produktionskosten und niedrigere Gewinne durch alle anderen Aktionäre und die Stakeholder mitbezahlt.

Zusätzlich eröffnen die Finanzmärkte in der heutigen Zeit kaum überblickbare Kanäle der „Umwegplünderung“. Anabtawi (2006, 31-32) berichtet über den Hedge Fund Perry Capital, der 2004 einen Aktienblock der Mylan Laboratories erwarb und sich dabei gleichzeitig gegen das Kursrisiko durch einen Derivatvertrag mit Hilfe entsprechender Put-Optionen vollständig absicherte. Als grosser Mylan-Blockhalter übte Perry dann Druck aus, dass Mylan die Firma King Pharmaceuticals mit einem signifikanten Aufschlag über dem Marktpreis erwerben sollte. Von der Überzahlung für King profitierte der Hedge Fund Perry, weil er gleichzeitig einen grossen Aktienblock an dem Übernahmekandidaten hielt. Der Kursverlust der Mylan-Aktien nach der Bekanntgabe der Übernahmeabsichten liess Perry wegen seiner Put-Optionen hingegen kalt. Andere Aktionäre, aber auch Mitarbeiter, werden in solchen Fällen von den Umwegprofiteuren systematisch geschädigt. Die vielfältigen Möglichkeiten der Investoren, durch Derivatverträge derartige Kanäle der Umwegrentabilität gezielt zu konstruieren, lassen die Idee homogener Shareholderinteressen weltfremd erscheinen.

\subsection{Das Problem der Mitarbeiterpolitik}

Grundsätzlich gelten Mitarbeiter als eine Stakeholder-Gruppe mit potentiell hohen Interessendivergenzen, da sich ihre Aufgaben, Rollen und Lebenssituationen entlang unabsehbar vielen Dimensionen unterscheiden: Alter, Geschlecht, Ausbildung, Gesundheit, familiäre Einbindung, familiäre Mobilität, Arbeitsort, Funktion usw., um nur einige zu nennen (vgl. hierzu und zum Folgenden ausführlich Hansmann 1993, 535). Verschiedene Mitarbeiter oder Gruppen von Mitarbeitern sind daher von bestimmten Entscheidungen der Unternehmensführung unterschiedlich betroffen und bewerten die Entscheidungsfolgen dementsprechend anders. Wie Hansmann (1993) gezeigt hat, sind heterogene Mitarbeiterinteressen sprichwörtlich schwer aggregierbar zu einem allgemein akzeptierten Standard, den die Be-

9 Der Begriff „Shareholder Sharking“ wurde von Stout $(2007,795)$ übernommen. 
teiligten selbst oder auch Dritte, wie z. B. Gerichte, in Konfliktsituationen zur Problemlösung anwenden könnten (Hansmann 1993, 593).

Als Fazit lässt sich festhalten, dass es erhebliche Interessendivergenzen zwischen Aktionären aufgrund der Blockhalter-Strukturen realer Aktiengesellschaften gibt. Auch zwischen den Mitarbeitern gibt es markante Interessendivergenzen. Hinzu kommt der Verteilungskonflikt zwischen Mitarbeitern und Aktionären. Angesichts der Logik der Teamproduktion, die eine Separierung der Einzelbeiträge der Teammitglieder zum Teamoutput nicht wirklich erlaubt, droht ein mit Interessenvertretern besetztes Führungsgremium der Aktiengesellschaft zur Arena der Rentensuche mit eskalierenden Politikkosten zu werden.

\section{Board Primacy als „Entwaffnungslösung“}

Vor diesem Hintergrund erscheint die im Aktienrecht eindeutig an die Direktoren zugewiesene Befehlsgewalt in einem anderen Lichte. ${ }^{10}$ Genau dadurch wird nämlich eine unabhängige dritte Partei geschaffen, die gerade keine Gruppeninteressen vertritt, sondern im Namen der Unternehmung als Ganzes agiert, wie Blair und Stout (1999, 291) herausarbeiten:

"In the eyes of the law, corporate directors are a unique form of fiduciary who, to the extent that they resemble any other form, perhaps most closely resemble trustees. Like trustees, directors, once elected, become the ultimate decision-making authority within the firm, constrained primarily by their fiduciary duties... American law in fact grants directors tremendous discretion to sacrifice shareholders' interests in favor of management, employees, and creditors, in deciding what is best for 'the firm'. "

Die gesetzlich verankerte Board Primacy bedeutet eben gerade keine Shareholder Primacy und auch keine Employee Primacy und daher nichts anderes als eine bewusste und gezielte „Abrüstung“ der spezifischen Investoren (Rajan/Zingales 1998; Blair/Stout 1999, 2001; Stout 2003). Genau wegen der Board Primacy können die unternehmensspezifischen Investoren die „Macht des Eigentums“ nicht mehr gegeneinander einsetzen. Die eigene Schutzlosigkeit, die man vorher durch die Aufrüstung mit Mitspracherechten zu heilen versuchte, wird aufgrund der Board Primacy irrelevant. Ihr wird durch die Nichtangriffsfähigkeit der anderen spezifischen Investoren die Brisanz genommen. Ein unabhängiger Treuhänder-Verwaltungsrat schafft Anreize für unternehmensspezifische Investoren, weil er die politische Rentensuche unterbindet. ${ }^{11}$

\section{Die Marktsignale der Aktionäre}

Bis jetzt wurde ausschliesslich auf der Grundlage von Theorien argumentiert. Es ist höchste Zeit, die Frage zu stellen, wie sich die Aktionäre selbst zu dieser Debatte äussern. Glaubhafte Äusserungen der Aktionäre bleiben an den Grundsatz „to put their money where their mouths are“ (Stout 2007, 805-806) verwiesen. Die Frage lautet daher: Wie stimmen die

10 Es ist bezeichnend, dass Art. 716a OR (und damit eine besonders ausgeprägte Board Primacy) in der Schweiz mit der Aktienrechtsrevision von 1991 eingeführt wurde. Es war das Ziel, die Benachteiligung der Minderheitsaktionäre durch die kontrollierenden Aktionäre zu beheben. Diesen Hinweis, den ich als „Board Primacy gegen Shareholder Sharking“ interpretiere, verdanke ich ebenfalls Hans Caspar von der Crone.

11 Oder wie Stout $(2007,797)$ ausführt: „Board governance, as a result, attracts specific investors by offering... the promise of 'business continuity'." 
Aktionäre im Rahmen ihrer Investitionsentscheidungen, also beim Aktienkauf selbst, über die Stärkung ihrer Rechte ab?

Aktionärsrechte können in vielen Ländern freiwillig und ohne jeden gesetzlichen Zwang in die Statuten aufgenommen werden. Dies geht z. B. in den USA noch viel leichter als in der Schweiz, wo ein besonderer Schutz für die undelegierbaren und unentziehbaren Aufgaben des Verwaltungsrats gilt. Firmen könnten sich also durch aktionärsfreundlichere Statuten einen Wettbewerbsvorteil verschaffen, wenn die Aktionäre tatsächlich glaubten, von einer Stärkung der Aktionärsrechte zu profitieren. Stout $(2007,802)$ bringt es wie folgt auf den Punkt:

„If investors truly believed greater shareholder control meant better corporate performance, they could "vote with their wallets" by preferring shares in firms that give sharebolders more control."

Reale Aktionäre scheinen dies aber nicht zu glauben. Gleich mehrere empirische Studien (Casares Field/Karpoff 2002; Coates 2001; Daines/Klausner 2001) befassen sich z. B. mit der Statutengestaltung bei Firmen, die erstmals an die Börse gehen in einem so genannten Initial Public Offering (IPO). Ihre Anreize, Governance-Regeln anzubieten, die bei externen Investoren gut ankommen, liegen auf der Hand. Wie Stout (2007, 802-803) in seiner Kommentierung dieser Studien feststellt, führt das Eingehen auf die Bedürfnisse der Investoren zuverlässig in die Gegenrichtung: Die Firmen nutzen die Statuten gezielt, um die Aktionärsrechte weiter einzuschränken. Entsprechend resümiert Stout (2007, 803):

„Are investors stupid? Why do they not avoid IPOs with weak shareholder rights? Is it possible that shareholders, like Ulysses, sometimes see advantage in ,tying their own hands" and ceding control over the corporation to directors largely insulated from their own influence?"

In einer ganz aktuellen empirischen Studie aus den USA untersuchen Larcker et al. (2010) die Reaktion des Kapitalmarktes auf 18 verschiedene gesetzliche und regulatorische Eingriffe in die Corporate Governance, die zwischen März 2007 und Juni 2009 stattfanden. Sie gruppieren die 18 Massnahmen in zwei nicht exklusive Kategorien: „Executive Pay Events“ und „Proxy Access Events“. Wie würde der Kapitalmarkt darauf reagieren, dass den Aktionären in den von der Regulierung betroffenen Firmen mehr Mitsprache über die Direktorenentlohnung und/oder mehr Einfluss auf das Nominierungs- und Wahlverfahren eingeräumt wird? Auf der Basis von 46.683 Firmen-Tages-Beobachtungen kommen die Autoren zu folgendem Fazit (Larcker et al. 2010, 34):

„Across all tests, we find generally robust evidence of negative stock price reactions for firms whose governance practices would be affected by the proposed regulations. The results support the notion that the proposed governance regulations harm the shareholders of the affected firms."

Offenbar stimmen die Aktionäre auch ausserhalb von IPOs mit dem Portemonnaie regelmässig gegen eine Stärkung ihrer Rechte ab. Wagner und Wenk (2011) publizierten kürzlich eine Studie über die Reaktion des Schweizer Aktienmarktes auf die Einreichung der MinderInitiative mit 115.000 Unterschriften am 26. Februar 2008. Die klaren Negativreaktionen bestätigen das Bild aus den USA. Auch die Aktionäre der Schweizer Firmen scheinen wenig 
davon begeistert, über mehr Rechte etwa einen bindenden Einfluss über die Entlohnungspolitik ausüben zu können.

Wie sind die Forderungen der Shareholder-Aktivisten zu interpretieren, die im Widerspruch zu diesem beobachteten Investitionsverhalten stehen? Einige Autoren erkennen darin lediglich eine Geschäftsstrategie grosser Investoren bzw. professioneller Aktionärsvertreter (vgl. z. B. Anabtawi 2006; Bainbridge 2006, 1751-1757; Stout 2007, 805-807). Diese haben vielfältige Möglichkeiten der Rentensuche aufgrund real- oder finanzwirtschaftlicher Umwege. Das einzige, was sie daran hindert, existierende Aktiengesellschaften zur Verfolgung der eigenen Geschäftspolitik auf Kosten der anderen Stakeholder zu instrumentalisieren, sind starke und unabhängige Boards. Für diese Akteure ist Shareholder-Aktivismus daher ein Instrument, um Boards gefügig zu machen. So schreibt Anabtawi (2006, 23-24) über den Hedge-Fund Betreiber Carl Icahn:

„In communicating with other shareholders, Icahn typically uses as his mantra the claim of wanting to make sure that the board is 'focused on maximizing shareholder value'. However, the short term investment horizon of hedge funds and Icahn's history of reaching 'peaceful' solutions with existing management that involve greenmail-type payments and other substantial concessions to him, raises questions about whether such hedge fund activism benefits shareholders in the long run."

\section{Fazit}

Das Konzept der Corporate Governance, bei der Aktionäre zu Eigentümern der Aktiengesellschaft, zu den einzigen Haltern von Residualansprüchen und zu Prinzipalen der Direktoren erhoben werden, hat viele Anhänger. ${ }^{12}$ Unabhängig von seinem Wert als theoretisches Verfassungskonstrukt, erfasst es die institutionelle Realität der Aktiengesellschaft nicht.

Erstens sind die Aktionäre keine Eigentümer der Aktiengesellschaft. Als unabhängige rechtliche Einheit „gehört“ die Aktiengesellschaft sich selbst. Aktionäre besitzen lediglich ein Wertpapier, das ihnen genau abgezählte und abgegrenzte Rechte einräumt (vgl. dazu ausführlich Stout 2007, 804). Zweitens sind die Aktionäre eine Stakeholdergruppe unter mehreren, die aufgrund geleisteter spezifischer Investitionen an dem „übrig gebliebenen“ Risiko der Unternehmung beteiligt sind. Drittens sind die Aktionäre keineswegs die Prinzipale und die Direktoren deren Agenten. Die Direktoren sind eindeutig die Letztentscheidungsinstanz der Aktiengesellschaft. Sie haben die treuhänderische Freiheit, Interessen der Aktionäre zu Gunsten anderer Stakeholder hintanzustellen, sofern dies dem Wohle der Unternehmung dient.

Die theoretische Analyse legt nahe, dass diese institutionellen Weichenstellungen nicht zufällig getroffen wurden. Es gibt gute Gründe, daran festzuhalten und die Board Primacy nicht zu gefährden. ${ }^{13}$ Umso mehr erstaunt es, dass die häufig aus dem Kreis grosser Investoren lancierten Forderungen nach mehr „Aktionärsdemokratie“ so breite mediale und gesellschaftliche Akzeptanz finden, gerade auch in jenen Kreisen der Bevölkerung, die gar nicht an Börsen spekulieren. Der Grund liegt wohl in dem verbreiteten Gefühl, dass angesichts der

12 Der Autor gehörte auch längere Zeit dazu.

13 Sehr deutlich machen dies auch Vogt et al. (2009, 1385f.) in ihrem juristischen Beitrag zur Aktienrechtsrevision in der Schweiz. 
Finanzskandale und Salärexzesse bei grossen Aktiengesellschaften endlich „etwas passieren muss". 14

Studiert man aber die Krisenfälle wie z. B. Enron etwas genauer, so wird man überrascht feststellen, unter den selbst erklärten „Musterschülern“ des Shareholder Value-Denkens gelandet zu sein. ${ }^{15}$ Enron-Direktoren waren über umfangreiche Aktienoptionsprogramme besonders stark angereizt, den Shareholder Value zu maximieren. Bei Enron waren die Aktionäre also über den Umweg des Entlohnungssystems zu Quasi-Prinzipalen mutiert und die Direktoren zu deren Quasi-Agenten. Die gesetzlich verankerte Board Primacy ermöglicht es dem Board natürlich auch, freiwillig in die Rolle eines „verlängerten Arms der Aktionäre“ zu schlüpfen. Diese inszenierte Shareholder Primacy, bei der sich der Verwaltungsrat als reiner Interessenvertreter der Aktionäre gebärdet, hat nicht wirklich funktioniert.

Daraus folgt aber keineswegs, dass nun die echte Shareholder Primacy mit Hilfe des Gesetzes umgesetzt werden müsste. Wenn man Gegensteuer geben möchte, sollte man sich besser darauf besinnen, was Board Primacy wirklich bedeutet und die Corporate Governance daran messen, ob der Verwaltungsrat tatsächlich die Rolle des Treuhänders zum Wohle der Unternehmung spielen kann. Das Treuhänder-Modell ist an Voraussetzungen gebunden:

Weder sollten Treuhänder-Verwaltungsräte Aktien oder Optionen halten, noch auf andere Weise direkt an der „Performance“ der Firma partizipieren. Leistungsanreize würden sie nämlich zu „Verbündeten“ einzelner Gruppen von Residualanspruchshaltern machen und ihre Fähigkeit „trüben“, zwischen konkurrierenden Interessen abzuwägen und zum Wohle der Firma als Ganzes zu entscheiden. Nur wer selbst kein „verlängerter Arm“ einer Interessengruppe ist, kann glaubwürdig das System der „Entwaffnung“ der Interessengruppen symbolisieren (Blair/Stout 2001, 443).

Allein über die Reputation auf dem Markt für Treuhänderdienste sollte die Verbindung zwischen dem erzielten Einkommen und dem Arbeitseinsatz des Verwaltungsrats verlaufen. Treuhänder, die residuale Kontrolle über längere Zeiträume in prosperierenden Firmen ausüben, stellen unter Beweis, dass sie den Mehrwert auf eine Weise zu verteilen in der Lage sind, die effiziente Ansätze für spezifische Investitionen schafft. Sie verbessern dadurch ihre Aussichten, erneut und zu einem höheren fixen Salär als Treuhänder bestellt zu werden (Fama/Jensen 1983a, 315).

Nicht „high powered incentives“ für Direktoren, wie es das Shareholder Value-Modell empfiehlt, sind der Schlüssel zu einem funktionierenden Verwaltungsrat, sondern „... a modest personal interest in ensuring the company's success and (perhaps more importantly) no strong financial interest in trying to expropriate wealth from other participants " (Stout 2007, 789).

\section{Literaturhinweise}

Alchian, A.A./Demsetz, H. (1972): Production, Information Costs, and Economic Organization, in: American Economic Review, Vol. 62, S. 777-795.

Anabtawi, I. (2006): Some Skepticism about Increasing Shareholder Power, in: UCLA Law Review, Vol. 53, unter http://cdn.law.ucla.edu/SiteCollectionDocuments/workshops\%20and\%20collo-

14 Stout $(2007,806)$ spricht vom „Enron effect“, der zur verbreiteten Ansicht führe, dass „something must be done".

15 Vgl. auch dazu Stout (2007, 806-807). Eine umfassende Analyse des Falls unter dem Titel „Enron and the Dark Side of Shareholder Value“ findet sich bei Bratton (2002). 
quia/some\%20skepticism\%20about\%20increasing\%20shareholder\%20power.pdf (abgefragt am 10.2.2011).

Bainbridge, S.M. (2006): Director Primacy and Shareholder Disempowerment, in: Harvard Law Review, Vol. 119, S. 1735-1758.

Bebchuk, L.A. (2005): The Case for Increasing Shareholder Power, in: Harvard Law Review, Vol. 118, S. 833-917.

Berle, A.A./Means, G.C. (1932): The Modern Corporation and Private Property, New York.

Bhagat, S./Blair, M.M./Black, B.S. (2004): Relational Investing and Firm Performance, in: Journal of Financial Research, Vol. 27, S. 1-30.

Black, B.S. (1990): Shareholder Passivity Reexamined, in: Michigan Law Review, Vol. 89, S. 520-608.

Blair, M.M. (1995): Ownership and Control, Washington D.C.

Blair, M.M. (1996): Wealth Creation and Wealth Sharing, Washington D.C.

Blair, M.M. (1999): Firm-Specific Human Capital and Theories of the Firm. Working Paper Nr. 167848, Georgetown University Law Center, unter http://papers.ssrn.com/sol3/Delivery.cfm/ SSRN_ID167848_code030912570.pdf?abstractid=167848\&mirid=1 (abgefragt am 10.2.2011).

Blair, M.M./Stout, L.A. (1999): A Team Production Theory of Corporate Law, in: Virginia Law Review, Vol. 85, S. 247-328.

Blair, M.M./Stout, L.A. (2001): Director Accountability and the Mediating Role of the Corporate Board, in: Washington University Law Quarterly, Vol. 79, S. 403-447.

Bratton, W.W. (2002): Enron and the Dark Side of Shareholder Value. Working Paper Nr. 035, Law School, George Washington University, unter http://papers.ssrn.com/sol3/Delivery.cfm/SSRN_ID301475_code020302630.pdf?abstractid=301475\&mirid=1 (abgefragt am 10.2.2011).

Bratton, W.W./Wachter, M.L. (2010): The Case against Shareholder Empowerment, in: University of Pennsylvania Law Review, Vol. 158, S. 653-728.

Brink, A. (2011): Spezifische Investitionen als Legitimationsgrundlage für Stakeholderansprüche, in: Die Unternehmung, Jg. 65, S. 50-68.

Coates, J.C. (2001): Explaining Variation in Takeover Defenses: Blame the Lawyers, in: California Law Review, Vol. 89, S. 1301-1421.

Casares Field, L./Karpoff, J. (2002): Takeover Defenses of IPO Firms, in: Journal of Finance, Vol. 57, S. 1857-1889.

Daines, R./Klausner, M. (2001): Do IPO Charters Maximize Firm Value? Antitakeover Protections in IPOs, in: Journal of Law, Economics and Organization, Vol. 83, S. 83-120.

Davis, G.F. (2009): The Rise and Fall of Finance and the End of the Society of Organizations, in: Academy of Management Perspectives, Vol. 23, S. 27-44.

Fama, E./Jensen M.C. (1983a): Separation of Ownership and Control, in: Journal of Law and Economics, Vol. 26, S. 301-325.

Fama, E./Jensen M.C. (1983b): Agency Problems and Residual Claims, in: Journal of Law and Economics, Vol. 26, S. 327-349.

Fauver, L./Fuerst, M.E. (2006): Does Good Corporate Governance Include Employee Representation? Evidence from German Corporate Boards, in: Journal of Financial Economics, Vol. 82, S. 673-710.

Franck, E. (2002): Zur Verantwortung des Verwaltungsrates aus ökonomischer Sicht, in: Die Unternehmung, Jg. 56, S. 213-225.

Franck, E. (2011): Ist es an der Zeit, die Aktionärsrechte zu stärken? Rede des Prorektors gehalten am Dies academicus 2011, Zürcher Universitätsschriften Nr. 13, Universität Zürich. 
Furubotn, E.G. (1988): Codetermination and the Modern Theory of the Firm: A Property-Rights Analysis, in: Journal of Business, Vol. 61, S. 165-181.

Hansmann, H. (1993): Worker Participation and Corporate Governance, in: University of Toronto Law Journal, Vol. 43, S. 589-606.

Hirschman, A.O. (1970): Exit, Voice, and Loyalty, Cambridge.

Initiativkomitee (2011): Eidgenössische Volksinitiative <<gegen die Abzockerei>>, unter http:// www.volksinitiative-gegen-die-abzockerei.ch/ (abgefragt am 10.2.2011).

Jacobson, L./LaLonde, R.J./Sullivan, D.G. (1993): Earnings Losses of Displaced Workers, in: American Economic Review, Vol. 83, S. 685-709.

Jensen, M.C. (2000): The Theory of the Firm, Cambridge.

Jensen, M.C./Meckling, W.H. (1976): Theory of the Firm: Managerial Behavior, Agency Costs, and Ownership Structure, in: Journal of Financial Economics, Vol. 3, S. 305-360.

Larcker, D.F./Ormazabal, G./Taylor, D.J. (2010): The Market Reaction to Corporate Governance

Regulation, in: Journal of Financial Economics (im Erscheinen), unter http://papers.ssrn.com/sol3/ Delivery.cfm/SSRN_ID1697324_code597368.pdf?abstractid=1650333\&mirid=1 (abgefragt am 10.2.2011).

Osterloh, M./Frey, B. (2006): Shareholders Should Welcome Workers as Directors, in: Journal of Management and Governance, Vol. 10, S. 325-345.

Rajan, R.G./Zingales, L. (1998): Power in a Theory of the Firm, in: Quarterly Journal of Economics, Vol. 113, S. 387-432.

Stadelmann, J. (2010): zRating: Corporate Governance in kotierten Schweizer Small \& Mid CapUnternehmen 2010. Empirische Studie von zCapital, Zug.

Stout, L.A. (2003): The Shareholder as Ulysses: Some Empirical Evidence on Why Investors in Public Corporations Tolerate Board Governance, in: University of Pennsylvania Law Review, Vol. 152, S. 1-47.

Stout, L.A. (2007): The Mythical Benefits of Shareholder Control, in: Virginia Law Review, Vol. 93, S. 789-809.

Topel, R.H. (1990): Specific Capital and Unemployment: Measuring the Costs and Consequences of Job Loss, in: Carnegie-Rochester Conference Series on Public Policy, Vol. 33, S. 181-214.

Vogt, H.-U./Schiwow, E./Wiedmer, K. (2009): Die Aktienrechtsrevision unter Corporate-GovernanceAspekten, in: AJP/PJA - die Zeitschrift für die Aktuelle Juristische Praxis, Jg. 11, S. 1359-1388.

Wagner, A./Wenk, C. (2011): Design Matters: Binding Say-on-Pay and Its Impact on Shareholder Value. Working Paper, April 2011, Department of Banking and Finance, University of Zurich.

Egon Franck ist ordentlicher Professor für Unternehmensführung und -politik an der Universität Zürich.

Anschrift: Universität Zürich, Institut für Betriebswirtschaftslehre, Plattenstrasse 14, CH-8032 Zürich, Tel.: +41(0)44/634-28-45, E-Mail: egon.franck@business.uzh.ch 